\title{
THE BI-EMBEDDABILITY RELATION FOR FINITELY GENERATED GROUPS II
}

\author{
SIMON THOMAS AND JAY WILLIAMS
}

Abstract. We study the isomorphism and bi-embeddability relations on the spaces of Kazhdan groups and finitely generated simple groups.

\section{IntroduCtion}

Let $\leq_{T}$ be the Turing reducibility relation on $2^{\mathbb{N}}$ and let $\equiv_{T}$ be the corresponding Turing equivalence relation. Then a classical result of Martin $[15,16]$ says that if $\mathcal{Z}$ is a $\equiv{ }_{T}$-invariant Borel subset of $2^{\mathbb{N}}$, then either $\mathcal{Z}$ or $2^{\mathbb{N}} \backslash \mathcal{Z}$ contains a cone with respect to Turing reducibility; i.e. a subset of the form $\mathcal{C}_{A_{0}}=\left\{A \in 2^{\mathbb{N}} \mid A_{0} \leq{ }_{T} A\right\}$ for some $A_{0} \in 2^{\mathbb{N}}$. It is natural to ask whether the corresponding result holds for the bi-embeddability relation $\approx_{e m}$ on the Polish space $\mathcal{G}_{f g}$ of finitely generated groups. A striking result of Friedman [7] implies that if $\mathcal{Z}$ is a $\approx_{e m}$-invariant Borel subset of $\mathcal{G}_{f g}$, then either $\mathcal{Z}$ or $\mathcal{G}_{f g} \backslash \mathcal{Z}$ is cofinal with respect to embeddability. (Here a subset $\mathcal{Z} \subseteq \mathcal{G}_{f g}$ is said to be cofinal with respect to embeddability if for every $G \in \mathcal{G}_{f g}$, there exists $H \in \mathcal{Z}$ such that $G \hookrightarrow H$.) However, in this paper, we will exhibit two natural examples $\mathcal{Z}$ of $\approx_{e m}$-invariant Borel subsets of $\mathcal{G}_{f g}$ such that neither $\mathcal{Z}$ or $\mathcal{G}_{f g} \backslash \mathcal{Z}$ contains a cone with respect to group embedding; i.e. a subset of the form $\mathcal{C}_{G_{0}}=\left\{G \in \mathcal{G}_{f g} \mid G_{0} \hookrightarrow G\right\}$ for some $G_{0} \in \mathcal{G}_{f g}$.

Remark 1.1. If $G_{0}, G_{1} \in \mathcal{G}_{f g}$, then $\mathcal{C}_{G_{0} \times G_{1}} \subseteq \mathcal{C}_{G_{0}} \cap \mathcal{C}_{G_{1}}$; in other words, the intersection of two cones contains a cone. More generally, the Higman-NeumannNeumann Embedding Theorem [12] implies that the intersection of countably many cones contains a cone.

For our first example, let $\mathcal{G}_{k a z}=\left\{K \in \mathcal{G}_{f g} \mid K\right.$ is a Kazhdan group $\}$. Then, by Shalom [20], $\mathcal{G}_{k a z}$ is an open subset of $\mathcal{G}_{f g}$; and since $\approx_{e m}$ is a countable Borel 
equivalence relation on $\mathcal{G}_{f g}$, it follows that

$$
\mathcal{K}=\left\{H \in \mathcal{G}_{f g} \mid H \approx_{e m} K \text { for some } K \in \mathcal{G}_{\text {kaz }}\right\}
$$

is a Borel subset of $\mathcal{G}_{f g}$.

Theorem 1.2. Both $\mathcal{K}$ and $\mathcal{G}_{f g} \backslash \mathcal{K}$ are cofinal with respect to embeddability; and hence neither contains a cone.

In [18], Ol'shanskii proved that the class of Kazhdan groups is cofinal with respect to embeddability; and so if the class of Kazhdan groups was closed under bi-embeddability, then Theorem 1.2 would follow easily. However, this is not the case.

Theorem 1.3. The subspace $\mathcal{G}_{k a z}$ of Kazhdan groups is not closed under biembeddability.

Of course, if $K$ is a Kazhdan group which is bi-embeddable with a non-Kazhdan group, then $K$ is necessarily non-cohopfian. (Recall that a group $G$ is said to be cohopfian if $G$ is not isomorphic to any of its proper subgroups.) The first examples of non-cohopfian Kazhdan groups were only recently discovered by Ollivier-Wise [17], de Cornulier [6] and Belegradek-Osin [2]. The proof of Theorem 1.3 involves the construction of a new class of non-cohopfian Kazhdan groups, based on the techniques of Higman-Scott [13] and Ol'shanskii [18]. During the course of proving Theorem 1.2, we will also obtain the following result.

Theorem 1.4. The bi-embeddability relation on the space $\mathcal{G}_{k a z}$ of Kazhdan groups is a weakly universal countable Borel equivalence relation.

Theorem 1.4 does not allow us to deduce anything about the Borel complexity of the isomorphism relation $\cong$ on the space $\mathcal{G}_{k a z}$ of Kazhdan groups. (For example, in [25], Williams constructed a subspace $\mathcal{X}$ of the space $\mathcal{G}_{f g}$ of finitely generated groups such that $\cong \uparrow \mathcal{X}$ is smooth and $\approx_{e m}\lceil\mathcal{X}$ is countable universal.) However, in Section 4, making use of results of Gao [8] and Shalom [21], we will prove the following result.

Theorem 1.5. The isomorphism relation on the space $\mathcal{G}_{\text {kaz }}$ of Kazhdan groups is a weakly universal countable Borel equivalence relation. 
Conjecture 1.6. The isomorphism and bi-embeddability relations on the space $\mathcal{G}_{k a z}$ of Kazhdan groups are universal countable Borel equivalence relations.

For our second example, let $\mathcal{G}_{\text {sim }}=\left\{K \in \mathcal{G}_{f g} \mid K\right.$ is a simple group $\}$. Since the class of simple groups is axiomatizable by an $\mathcal{L}_{\omega_{1} \omega}$-sentence, it follows that $\mathcal{G}_{\text {sim }}$ is a Borel subset of $\mathcal{G}_{f g}$; and this implies that

$$
\mathcal{S}=\left\{H \in \mathcal{G}_{f g} \mid H \approx_{e m} G \text { for some } G \in \mathcal{G}_{\text {sim }}\right\}
$$

is a Borel subset of $\mathcal{G}_{f g}$.

Theorem 1.7. Both $\mathcal{S}$ and $\mathcal{G}_{f g} \backslash \mathcal{S}$ are cofinal with respect to embeddability; and hence neither contains a cone.

It is well-known that the subspace $\mathcal{G}_{\text {sim }}$ of finitely generated simple groups is not closed under bi-embeddability. For example, let $V$ be Thompson's larger finitely presented infinite simple group. Then, regarding $V$ as the group of dyadic homeomorphisms of the Cantor space, it is clear that $V \times V \hookrightarrow V$. (For a clear account of the basic properties of the Thompson groups, see Cannon-Floyd-Parry [4].) In [23], Thomas proved that the isomorphism relation on $\mathcal{G}_{\text {sim }}$ is not smooth; and, since $\left(\cong\left\lceil\mathcal{G}_{\text {sim }}\right) \subseteq\left(\approx_{\text {em }} \mid \mathcal{G}_{\text {sim }}\right)\right.$, this implies that the bi-embeddability relation on $\mathcal{G}_{\text {sim }}$ is also not smooth. (For example, see Thomas [23, Proposition 2.1].) As we will see in Section 3, it is not possible to modify the proof of Theorem 1.4 so that it applies to the space $\mathcal{G}_{\text {sim }}$ of finitely generated simple groups.

Conjecture 1.8. The isomorphism and bi-embeddability relations on the space $\mathcal{G}_{\text {sim }}$ of finitely generated simple groups are universal countable Borel equivalence relations.

Acknowledgements: We would like to thank Ilia Kapovitch, Jesse Peterson and Chuck Weibel for helpful discussions concerning the material in this paper. We are especially grateful to Jesse Peterson for supplying Theorem 4.2.

\section{Preliminaries}

In this section, we will recall some basic notions and results from group theory, recursion theory and the theory of countable Borel equivalence relations. 
2.1. Group Theory. Throughout this paper, $\mathcal{G}_{f g}$ denotes the Polish space of finitely generated groups introduced by Grigorchuk [9]; i.e., the elements of $\mathcal{G}_{f g}$ are the isomorphism types of marked groups $\langle G, \bar{c}\rangle$, where $G$ is a finitely generated group and $\bar{c}$ is a finite sequence of generators. (For a clear account of the basic properties of the space $\mathcal{G}_{f g}$, see either Champetier [5] or Grigorchuk [10].) Following the usual convention, we will slightly abuse notation and denote the elements of $\mathcal{G}_{f g}$ by $G, H$, etc. instead of the more accurate $(G, \bar{c}),(H, \bar{d})$, etc.

Definition 2.1. If $G$ is a (not necessarily finitely generated) group, then the corresponding skeleton is $\operatorname{Sk}(G)=\left\{H \in \mathcal{G}_{f g} \mid H \hookrightarrow G\right\}$.

Throughout this paper, by a Kazhdan group, we will always mean a countable discrete Kazhdan group. The following result collects together the properties of Kazhdan groups that are needed in the proofs of Theorems 1.2, 1.3 and 1.4. (A clear account of the theory of Kazhdan groups can be found in Bekka-de la HarpeValette [1].)

Theorem 2.2. Let $K$ be a countable discrete Kazhdan group.

(i) $K$ is finitely generated.

(ii) If $N \unlhd K$, then $K / N$ is also a Kazhdan group.

(iii) If $K$ is amenable, then $K$ is finite.

2.2. Recursion Theory. Throughout this paper, the powerset $\mathcal{P}(\mathbb{N})$ will be identified with the Cantor space $2^{\mathbb{N}}$ by identifying each subset $A \subseteq \mathbb{N}$ with its characteristic function $\chi_{A} \in 2^{\mathbb{N}}$. Let $\operatorname{Fin}(\mathbb{N})$ be the collection of finite subsets of $\mathbb{N}$. If $A$, $B \subseteq \mathbb{N}$, then $A$ is said to be enumeration reducible to $B$, written $A \leq_{e} B$, if there exists a recursively enumerable subset $W \subseteq \mathbb{N} \times \operatorname{Fin}(\mathbb{N})$ such that

$$
n \in A \quad \Longleftrightarrow \quad(n, F) \in W \text { for some finite subset } F \subseteq B
$$

Intuitively, $A$ is enumeration reducible to $B$ if there is an effective procedure which produces an enumeration of $A$ from any enumeration of $B$. Let $\equiv_{e}$ be the corresponding countable Borel equivalence relation on $2^{\mathbb{N}}$ defined by

$$
A \equiv_{e} B \quad \Longleftrightarrow \quad A \leq_{e} B \text { and } B \leq_{e} A .
$$

Then for each $A \in 2^{\mathbb{N}}$, the corresponding e-degree is $\mathbf{a}=\left\{B \in 2^{\mathbb{N}} \mid B \equiv_{e} A\right\}$. 
For the basic theory of enumeration reducibility and a clear account of its many group theoretic connections, see Higman-Scott [13]. Here we will just review a few basic notions and results that will play a key role in the followings sections. For each $n \geq 1$, let $\left\{w_{k}\left(x_{1}, \cdots, x_{n}\right) \mid k \in \mathbb{N}\right\}$ be a fixed effective enumeration of the (not necessarily reduced) words in $x_{1}, \cdots, x_{n}, x_{1}^{-1}, \cdots, x_{n}^{-1}$. If $G=\left\langle a_{1}, \cdots, a_{n}\right\rangle$ is a finitely generated group, then we define

$$
\operatorname{Rel}(G)=\left\{k \in \mathbb{N} \mid w_{k}\left(a_{1}, \cdots, a_{n}\right)=1\right\}
$$

Of course, there is a slight abuse of notation here, since the set $\operatorname{Rel}(G)$ clearly depends on the sequence of generators $a_{1}, \cdots, a_{n}$. However, if $b_{1}, \cdots, b_{m}$ is any other sequence of generators of $G$, then it is easily seen that

$$
\left\{k \in \mathbb{N} \mid w_{k}\left(a_{1}, \cdots, a_{n}\right)=1\right\} \equiv{ }_{e}\left\{\ell \in \mathbb{N} \mid w_{\ell}\left(b_{1}, \cdots, b_{m}\right)=1\right\}
$$

In other words, the $e$-degree of $\operatorname{Rel}(G)$ is independent of the choice of generating set of $G$. It is well-known that if $R$ is a set of defining relations for the group $G=\left\langle a_{1}, \cdots, a_{n}\right\rangle$, then $\operatorname{Rel}(G) \leq_{e} R$; and that if $G \hookrightarrow H$, then $\operatorname{Rel}(G) \leq_{e} \operatorname{Rel}(H)$. Furthermore, for each $S \in 2^{\mathbb{N}}$, there exists a finitely generated group $A_{S}$ such that $\operatorname{Rel}\left(A_{S}\right) \equiv_{e} S$; and, in fact, the group $A_{S}$ can be chosen to be solvable. (For example, see the proof of Higman-Scott [13, Corollary 4.24].)

Definition 2.3. Let $S \in 2^{\mathbb{N}}$ and let $\mathbf{s}$ be the corresponding $e$-degree. Then the finitely generated group $G_{\mathbf{s}}$ is said to be relatively universal of degree $\mathbf{s}$ if

$$
\operatorname{Sk}\left(G_{\mathbf{s}}\right)=\left\{H \in \mathcal{G}_{f g} \mid \operatorname{Rel}(H) \leq_{e} S\right\}
$$

By Higman-Scott [13, Corollary 6.4], there exists a relatively universal group $G_{\mathbf{s}}$ of each $e$-degree $\mathbf{s}$; and in Section 3 , we will prove that $G_{\mathbf{s}}$ can be chosen to be a Kazhdan group. Of course, it is clear that if $G_{\mathbf{s}}, G_{\mathbf{s}}^{\prime}$ are both relatively universal of degree $\mathbf{s}$, then $G_{\mathbf{s}}, G_{\mathbf{s}}^{\prime}$ are bi-embeddable.

Remark 2.4. It is interesting to note that the analogous result fails for Turing reducibility; namely, for each $S \in 2^{\mathbb{N}}$, there does not exist a finitely generated group $G$ such that $\operatorname{Sk}(G)=\left\{H \in \mathcal{G}_{f g} \mid \operatorname{Rel}(H) \leq_{T} S\right\}$. To see this, it is enough to show that if $G$ is any finitely generated group, then there exists a finitely generated group $H$ such that: 
(a) $H$ does not embed into $G$; and

(b) $\operatorname{Rel}(H) \leq_{T} \operatorname{Rel}(G)$.

As we have not been able to find a reference for this result, we will sketch the proof. Let $G=\left\langle a_{1}, \cdots, a_{n}\right\rangle$ and let $\left\{\left\langle u_{k}\left(x_{1}, \cdots, x_{n}\right), v_{k}\left(x_{1}, \cdots, x_{n}\right)\right\rangle \mid k \in \mathbb{N}\right\}$ be an effective enumeration of the ordered pairs of words in $x_{1}, \cdots, x_{n}, x_{1}^{-1}, \cdots, x_{n}^{-1}$. For each $k \in \mathbb{N}$, let $r_{k}(x, y)$ be the word $\left(x^{k+1} y^{k+1}\right)^{7}$. Consider the finitely generated group $H$ with presentation $\left\langle b, c \mid\left\{r_{k}(b, c) \mid k \in I\right\}\right\rangle$, where

$$
I=\left\{k \in \mathbb{N} \mid r_{k}\left(u_{k}\left(a_{1}, \cdots, a_{n}\right), v_{k}\left(a_{1}, \cdots, a_{n}\right)\right) \neq 1\right\} .
$$

Clearly $H$ satisfies the $C^{\prime}(1 / 6)$ cancellation property; and since $I \leq_{T} \operatorname{Rel}(G)$, it follows that $\operatorname{Rel}(H) \leq_{T} \operatorname{Rel}(G)$. (See Lyndon-Schupp [14, Section V.4].) If $\varphi$ is an embedding of $H$ into $G$, then there exists $k \in \mathbb{N}$ such that $\varphi(b)=u_{k}\left(a_{1}, \cdots, a_{n}\right)$ and $\varphi(c)=v_{k}\left(a_{1}, \cdots, a_{n}\right)$. Clearly $k \notin I$ and so $r_{k}\left(u_{k}\left(a_{1}, \cdots, a_{n}\right), v_{k}\left(a_{1}, \cdots, a_{n}\right)\right)=1$. But since $k \notin I$, the $C^{\prime}(1 / 6)$ cancellation property implies that $r_{k}(b, c) \neq 1$, which is a contradiction.

2.3. Countable Borel Equivalence Relations. Let $X$ be a Polish space. Then a Borel equivalence relation on $X$ is an equivalence relation $E \subseteq X^{2}$ which is a Borel subset of $X^{2}$. If $E, F$ are Borel equivalence relations on the Polish spaces $X$, $Y$ respectively, then a Borel map $f: X \rightarrow Y$ is said to be a homomorphism from $E$ to $F$ if for all $x, y \in X$,

$$
x E y \quad \Longrightarrow \quad f(x) F f(y)
$$

If $f$ satisfies the stronger property that for all $x, y \in X$,

$$
x E y \quad \Longleftrightarrow \quad f(x) F f(y),
$$

then $f$ is said to be a Borel reduction and we write $E \leq_{B} F$.

The Borel equivalence relation $E$ on the Polish space $X$ is said to be countable if every $E$-class is countable. The least complex countable Borel equivalence relations with respect to Borel reducibility are those which are smooth; i.e. those countable Borel equivalence relations $E$ such that $E$ is Borel reducible to the identity relation $\operatorname{Id}_{Y}$ on some (equivalently every) uncountable Polish space $Y$. On the other hand, the most complex are those countable Borel equivalence relations $E$ 
which are universal in the sense that $F \leq_{B} E$ for every countable Borel equivalence relation $F$. For example, as we mentioned earlier, Williams [25] has shown that the bi-embeddability relation $\approx_{e m}$ on $\mathcal{G}_{f g}$ is a universal countable Borel equivalence relation. Finally, a countable Borel equivalence relation $E$ is said to be weakly universal if for every countable Borel equivalence relation $F$, there exists a countable-to-one Borel homomorphism from $F$ to $E$. For example, it is well-known that the Turing equivalence relation $\equiv_{T}$ is weakly universal. Furthermore, applying Rogers [19, Theorem XXIV], it follows that $\equiv_{T} \leq_{B} \equiv_{e}$ and hence $\equiv_{e}$ is also weakly universal. It is currently not known whether either $\equiv_{T}$ or $\equiv_{e}$ is universal; and, in fact, it is remains an open question whether every weakly universal countable Borel equivalence relation is universal.

\section{Relatively universal Kazhdan groups}

In this section, we will prove all of the results mentioned in the Introduction, except for Theorem 1.5 which will be proved in Section 4. Our approach is based upon the following strengthening of Higman-Scott [13, Corollary 6.4].

Theorem 3.1. Let $S \in 2^{\mathbb{N}}$ and let $\mathbf{s}$ be the corresponding e-degree. Then there exists a relatively universal Kazhdan group $K_{\mathbf{s}}$ of degree $\mathbf{s}$.

Before proving Theorem 3.1, we will first derive two easy consequences.

Corollary 3.2. If $G$ is any finitely generated group, then there exists a Kazhdan group $K$ such that $G \hookrightarrow K$.

Proof. Let $S=\operatorname{Rel}(G)$ and let $K=K_{\mathrm{s}}$ be a relatively universal Kazhdan group of degree $\mathbf{s}$.

Corollary 3.3. The class of Kazhdan groups is not closed under bi-embeddability.

Proof. Fix some $S \in 2^{\mathbb{N}}$ and let $K_{\mathbf{s}}$ be a relatively universal Kazhdan group of degree $\mathbf{s}$. Then clearly

$$
\operatorname{Rel}\left(K_{\mathbf{s}} \times \mathbb{Z}\right) \leq_{e} \operatorname{Rel}\left(K_{\mathbf{s}}\right) \equiv_{e} S
$$

Thus $K_{\mathbf{s}} \times \mathbb{Z} \hookrightarrow K_{\mathbf{s}}$ and so $K_{\mathbf{s}} \times \mathbb{Z} \approx_{e m} K_{\mathbf{s}}$. However, since the infinite abelian group $\mathbb{Z}$ is a homomorphic image of $K_{\mathrm{s}} \times \mathbb{Z}$, it follows that $K_{\mathrm{s}} \times \mathbb{Z}$ is not a Kazhdan group. 
Proof of Theorem 3.1. By Higman-Scott [13, Corollary 6.4], there exists a finitely generated relatively universal group $L$ of degree $\mathbf{s}$. Hence it is enough to show that there exists a Kazhdan group $K_{\mathbf{s}}$ such that $L \leqslant K_{\mathbf{s}}$ and $\operatorname{Rel}\left(K_{\mathbf{s}}\right) \leq_{e} S$.

Let $H$ be an infinite hyperbolic Kazhdan group. (For example, we can let $H$ be a co-compact lattice in $S p(n, 1)$ for some $n \geq 2$. See de la Harpe-Valette [11].) Suppose that $L$ is an $n$-generator group. Applying Ol'shanskii [18], there exists a free group

$$
F=\left\langle a_{1}, \cdots, a_{n}\right\rangle \leqslant H
$$

of rank $n$ such that for every normal subgroup $N \unlhd F$, there exists a normal subgroup $M \unlhd H$ such that $N=M \cap F$. Equivalently, for any subset $R \subseteq F$, the corresponding normal closures $\left\langle R^{F}\right\rangle,\left\langle R^{H}\right\rangle$ satisfy $\left\langle R^{F}\right\rangle=\left\langle R^{H}\right\rangle \cap F$.

Since $H$ is hyperbolic, it follows that $H$ is finitely presentable. (For example, see Bridson-Haefliger [3, Proposition III.Г.2.2].) Let $H=\left\langle x_{1}, \cdots, x_{m} \mid T\right\rangle$ be a finite presentation; and for each $1 \leq i \leq n$, express $a_{i}=u_{i}(\bar{x})$ as a word in the generators $x_{1}, \cdots, x_{m}$. Let $K_{\mathbf{s}}$ be the group with presentation $\left\langle x_{1}, \cdots, x_{m} \mid R\right\rangle$, where

$$
R=T \cup\left\{w\left(u_{1}(\bar{x}), \cdots, u_{n}(\bar{x})\right) \mid w\left(a_{1}, \cdots, a_{n}\right) \in \operatorname{Rel}(L)\right\} .
$$

Then clearly $\operatorname{Rel}\left(K_{\mathbf{s}}\right) \leq_{e} R \leq_{e} \operatorname{Rel}(L) \leq_{e} S$. Furthermore, letting $M$ be the normal closure of $\left\{w\left(u_{1}(\bar{x}), \cdots, u_{n}(\bar{x})\right) \mid w\left(a_{1}, \cdots, a_{n}\right) \in \operatorname{Rel}(L)\right\}$ in $H$, it follows that $K_{\mathbf{s}}=H / M$ and $L \cong F /(M \cap F)$. Since the class of Kazhdan groups is closed under taking quotients, it follows that $K_{\mathbf{s}}=H / M$ satisfies our requirements.

It is natural to ask which other group theoretic properties can realized by relatively universal groups. Of course, the proof of Corollary 3.3 shows that relatively universal groups cannot be cohopfian. More interestingly, the next result shows that relatively universal groups cannot be simple. (In the proof of Theorem 3.4, $\operatorname{Nonrel}(G)=\mathbb{N} \backslash \operatorname{Rel}(G)$.)

Theorem 3.4. Let $S \in 2^{\mathbb{N}}$ and let $\mathbf{s}$ be the corresponding e-degree. If the finitely generated group $G_{\mathbf{s}}$ is relatively universal of degree $\mathbf{s}$, then $G_{\mathbf{s}}$ is not simple.

Proof. Suppose that $G_{\mathbf{s}}$ is a simple relatively universal group of degree s. Then, arguing as in the proof of Kuznetsov's Theorem, it follows that $\operatorname{Nonrel}\left(G_{\mathbf{s}}\right) \leq_{e} S$. (The proof of Kuznetsov's Theorem can be found in Lyndon-Schupp [14, IV.3.6].) 
By Higman-Scott [13, Lemma 6.7], there exists a finitely generated group $H$ such that $\operatorname{Rel}(H) \leq_{e} S$ and $\operatorname{Nonrel}(H) \varliminf_{e} S$. But then $H \hookrightarrow G_{\mathbf{s}}$ and this implies that $\operatorname{Nonrel}(H) \leq_{e} \operatorname{Nonrel}\left(G_{\mathbf{s}}\right)$, which is a contradiction.

It is well-known that every countable group embeds into a finitely generated simple group. (For example, see Lyndon-Schupp [14, IV.3.5].) Thus Theorem 1.7 is an immediate consequence of the following result.

Corollary 3.5. If $G$ is any finitely generated group, then there exists a finitely generated group $H$ such that:

(i) $G \hookrightarrow H$; and

(ii) $H$ is not bi-embeddable with a finitely generated simple group.

Proof. Let $S=\operatorname{Rel}(G)$ and let $\mathbf{s}$ be the corresponding $e$-degree. If $H=G_{\mathbf{s}}$ is relatively universal of degree s, then $H$ satisfies our requirements.

Next note that Theorem 1.2 is an immediate consequence of Corollary 3.2 and the following result.

Proposition 3.6. If $G$ is any finitely generated group, then there exists a finitely generated group $H$ such that:

(i) $G \hookrightarrow H$; and

(ii) $H$ is not bi-embeddable with a Kazhdan group.

Proof. Let $S \in 2^{\mathbb{N}}$ be such that $\operatorname{Rel}(G)<_{e} S$ and let $A_{S} \in \mathcal{G}_{f g}$ be a solvable group such that $\operatorname{Rel}\left(A_{S}\right) \equiv_{e} S$. We claim that the direct product $H=G \times A_{S}$ satisfies our requirements. If not, then there exists a Kazhdan group $K$ such that $K \approx_{e m} H$. In particular, it follows that $\operatorname{Rel}(K) \equiv_{e} \operatorname{Rel}(H)$. Clearly we can suppose that $K \leqslant H=G \times A_{S}$. Let $\pi: G \times A_{S} \rightarrow A_{S}$ be the canonical projection. Since $K /(K \cap \operatorname{ker} \pi)$ is an amenable Kazhdan group, it follows that $[K: K \cap \operatorname{ker} \pi]<\infty$; and this easily implies that $\operatorname{Rel}(K \cap \operatorname{ker} \pi) \equiv_{e} \operatorname{Rel}(K)$. Since $K \cap \operatorname{ker} \pi \leqslant G$, it follows that $\operatorname{Rel}(K \cap \operatorname{ker} \pi) \leq_{e} \operatorname{Rel}(G)$ and hence $\operatorname{Rel}(K) \leq_{e} \operatorname{Rel}(G)$, which is a contradiction.

Finally we will sketch the proof of Theorem 1.4. For each $S \in 2^{\mathbb{N}}$, let $\mathbf{s}$ be the corresponding $e$-degree. Then, examining the proofs of Corollary 4.24 and Theorem 
6.3 of Higman-Scott [13], together with the proof of Theorem 3.1, we see that there exist Borel maps $S \mapsto A_{S} \mapsto G_{S} \mapsto K_{S}$ such that:

(i) $A_{S}$ is a finitely generated group such that $\operatorname{Rel}\left(A_{S}\right) \equiv_{e} S$.

(ii) $G_{S}$ is a relatively universal group of degree s such that $A_{S} \leqslant G_{S}$.

(iii) $K_{S}$ is a relatively universal Kazhdan group of degree s such that $G_{S} \leqslant K_{S}$.

It follows that the map $S \mapsto K_{S}$ is a Borel reduction from $\equiv_{e}$ to $\approx_{e m}$ and hence the bi-embeddability relation on the space $\mathcal{G}_{k a z}$ is weakly universal.

Remark 3.7. Alternatively, notice that since $\left(\cong \uparrow \mathcal{G}_{k a z}\right) \subseteq\left(\approx_{e m} \mathcal{G}_{k a z}\right)$, the identity $\operatorname{map} K \mapsto K$ is a weak Borel reduction from $\cong \uparrow \mathcal{G}_{k a z}$ to $\approx_{e m} \mathcal{G}_{k a z}$; and hence Theorem 1.4 is also an immediate consequence of Theorem 1.5.

\section{THE ISOMORPHISM RELATION FOR KAZHDAN GROUPS}

In this section, making use of results of Gao [8] and Shalom [21], we will prove that the isomorphism relation on the space $\mathcal{G}_{k a z}$ of Kazhdan groups is weakly universal. It should be pointed out that the basic strategy of our proof is based on that of Champetier [5, Théorème 1.1].

First note that the canonical action of $S L_{2}(\mathbb{Z})$ on $\mathbb{Z}^{2}$, defined by

$$
\left(\begin{array}{ll}
a & b \\
c & d
\end{array}\right) \cdot\left(\begin{array}{l}
r \\
s
\end{array}\right)=\left(\begin{array}{l}
a r+b s \\
c r+d s
\end{array}\right),
$$

induces an associated Borel action on the powerset $\mathcal{P}\left(\mathbb{Z}^{2}\right)$, defined by

$$
\gamma \cdot A=\{\gamma \cdot \mathbf{v} \mid \mathbf{v} \in A\}
$$

for $\gamma \in S L_{2}(\mathbb{Z})$ and $A \in \mathcal{P}\left(\mathbb{Z}^{2}\right)$. Let $E_{\infty}^{\prime}$ be the corresponding orbit equivalence relation.

Theorem 4.1 (Gao [8]). The orbit equivalence relation $E_{\infty}^{\prime}$ of the action of $S L(2, \mathbb{Z})$ on the powerset $\mathcal{P}\left(\mathbb{Z}^{2}\right)$ is a universal countable Borel equivalence relation.

Next let $x, y$ be indeterminates and let $R=\mathbb{Z}\left[x, y, x^{-1}, y^{-1}\right]$. Then $S L_{2}(\mathbb{Z})$ also acts as a group of automorphisms of the ring $R$ via

$$
\left(\begin{array}{ll}
a & b \\
c & d
\end{array}\right) \cdot x^{r} y^{s}=x^{a r+b s} y^{c r+d s} .
$$

We are grateful to Jesse Peterson for providing us with the following key result. 
Theorem 4.2. There exists a Kazhdan group K such that:

(i) the center $Z(K)$ is the additive group of $R$; and

(ii) there is an embedding of $S L_{2}(\mathbb{Z})$ into $\operatorname{Aut}(K)$ which extends the above action of $S L_{2}(\mathbb{Z})$ on $R$.

Before proving Theorem 4.2, we will complete the proof that the isomorphism relation on the space $\mathcal{G}_{k a z}$ of Kazhdan groups is weakly universal. Consider the Borel map $f: \mathcal{P}\left(\mathbb{Z}^{2}\right) \rightarrow \mathcal{G}_{k a z}$ defined by $A \mapsto K / N_{A}$, where $N_{A}$ is the central subgroup of $K$ generated by $S_{A}=\left\{x^{a} y^{b} \mid\left(\begin{array}{c}a \\ b\end{array}\right) \in A\right\}$. Since the central elements $\left\{x^{a} y^{b} \mid\left(\begin{array}{l}a \\ b\end{array}\right) \in \mathbb{Z}^{2}\right\}$ freely generate $Z(K)$ as an abelian group, it follows that $f$ is injective. Furthermore, if $A, B \in \mathcal{P}\left(\mathbb{Z}^{2}\right)$ and $\gamma \in S L_{2}(\mathbb{Z})$ satisfies $\gamma \cdot A=B$, then the corresponding automorphism $\gamma \in \operatorname{Aut}(K)$ satisfies $\gamma\left[N_{A}\right]=N_{B}$ and so $K_{A} \cong K_{B}$. Thus $f$ is an injective Borel homomorphism from the countable universal Borel equivalence relation $E_{\infty}^{\prime}$ to $\cong \uparrow \mathcal{G}_{k a z}$; and it follows that $\cong \uparrow \mathcal{G}_{k a z}$ is weakly universal.

The remainder of this section will be devoted to the proof of Theorem 4.2. (Here we need to assume that the reader is familiar with the algebraic approach to Kazhdan groups developed by Shalom [21].) Let $R=\mathbb{Z}\left[x, y, x^{-1}, y^{-1}\right]$ and let $E L_{n}(R) \leqslant G L_{n}(R)$ be the subgroup generated by the elementary matrices over $R .^{1}$ Then it is easily checked that $R$ has Krull dimension 3. Hence, applying Shalom [21, Theorem 1.1], it follows that if $n \geq 5$, then $E L_{n}(R)$ is a Kazhdan group. Let $K$ be the subgroup of $G L_{7}(R)$ consisting of the matrices of the form

$$
\left(\begin{array}{ccc}
1 & \mathbf{k}_{1} & z \\
\mathbf{0} & A & \mathbf{k}_{2} \\
0 & \mathbf{0} & 1
\end{array}\right)
$$

where $A \in E L_{5}(R), \mathbf{k}_{1}$ is a $1 \times 5$ matrix, $\mathbf{k}_{2}$ is a $5 \times 1$ matrix and $z \in R$. Let $K_{1}$ and $K_{2}$ be the subgroups consisting of the matrices of the form

$$
\left(\begin{array}{ccc}
1 & \mathbf{k}_{1} & 0 \\
\mathbf{0} & I_{5} & 0 \\
0 & \mathbf{0} & 1
\end{array}\right),\left(\begin{array}{ccc}
1 & 0 & 0 \\
\mathbf{0} & I_{5} & \mathbf{k}_{2} \\
0 & \mathbf{0} & 1
\end{array}\right)
$$

\footnotetext{
${ }^{1}$ Although it is not needed in the following argument, it is perhaps worth mention that if $n \geq 5$, then $E L_{n}(R)=S L_{n}(R)$. (See Suslin [22] and Weibel [24, Chapter 3].)
} 
respectively; and let $\Gamma$ be the subgroup consisting of the matrices of the form

$$
\left(\begin{array}{lll}
1 & \mathbf{0} & 0 \\
\mathbf{0} & A & \mathbf{0} \\
0 & \mathbf{0} & 1
\end{array}\right) .
$$

Then $\Gamma$ normalizes $K_{1}$ and $K_{2}$; and for each $i=1,2$, we have

$$
K_{i} \rtimes \Gamma \cong R^{5} \rtimes E L_{5}(R)
$$

Hence, by Shalom [21, Theorem 2.4], for each $i=1,2$, the pair $\left(K_{i} \rtimes \Gamma, K_{i}\right)$ has the relative Kazhdan property; and, as mentioned above, $\Gamma \cong E L_{5}(R)$ is a Kazhdan group. Furthermore, $K$ is boundedly generated by $\left\{K_{1}, K_{2}, \Gamma\right\}$; in fact, it is easily checked that each $g \in K$ can be expressed as a product

$$
g=\gamma a_{1} b_{1} a_{2} b_{2} a_{2}^{-1} b_{2}^{-1}
$$

for some $\gamma \in \Gamma, a_{1}, a_{2} \in K_{1}$ and $b_{1}, b_{2} \in K_{2}$. Applying Shalom [21, Lemma 2.2], it follows that $K$ is a Kazhdan group. It is easily checked that that the center $Z(K)$ of $K$ consists of the matrices of the form

$$
\mathbf{z}=\left(\begin{array}{ccc}
1 & \mathbf{0} & z \\
\mathbf{0} & I_{5} & 0 \\
0 & \mathbf{0} & 1
\end{array}\right)
$$

for some $z \in R=\mathbb{Z}\left[x, y, x^{-1}, y^{-1}\right]$. Since each automorphism $\varphi \in \operatorname{Aut}(R)$ induces a corresponding automorphism $\left(a_{i j}\right) \mapsto\left(\varphi\left(a_{i j}\right)\right)$ of $K$, it is clear that $K$ satisfies our requirements.

Remark 4.3. It seems reasonable to expect that a more detailed analysis might show that the map $f: \mathcal{P}\left(\mathbb{Z}^{2}\right) \rightarrow \mathcal{G}_{k a z}$ (or perhaps some slight variant of $f$ ) is a Borel reduction from $E_{\infty}^{\prime}$ to $\cong \uparrow \mathcal{G}_{k a z}$; and hence that $\cong \uparrow \mathcal{G}_{k a z}$ is a universal countable Borel equivalence relation.

\section{REFERENCES}

[1] B. Bekka, P. de la Harpe and A. Valette, Kazhdan's property (T), Cambridge University Press, Cambridge, 2008.

[2] I. Belegradek and D. Osin, Rips construction and Kazhdan property (T), Groups, Geom. Dynam. 2 (2008), 1-12. 
[3] M. R. Bridson and A. Haefliger, Metric spaces of non-positive curvature, Springer-Verlag, Berlin, 1999.

[4] J. W. Cannon, W. J. Floyd and W. R. Parry, Introductory notes on Richard Thompson's groups, Enseign. Math. (2) 42 (1996), 215-256.

[5] C. Champetier, L'espace des groupes de type fini, Topology 39 (2000), 657-680.

[6] Y. de Cornulier, Finitely presentable, non-Hopfian groups with Kazhdan's property (T) and infinite outer automorphism group, Proc. Amer. Math. Soc. 135 (2007), 951-959.

[7] H. Friedman, New Borel independence results, preprint (2007).

[8] S. Gao, The action of $S L(2, \mathbb{Z})$ on the subsets of $\mathbb{Z}$, Proc. Amer. Math. Soc. 129 (2001), $1507-1512$.

[9] R. I. Grigorchuk, Degrees of growth of finitely generated groups and the theory of invariant means, Math. USSR-Izv. 25 (1985), 259-300.

[10] R. I. Grigorchuk, Solved and Unsolved Problems Around One Group, in Infinite Groups: Geometric, Combinatorial and Dynamical Aspects, Birkhäuser, Boston, 2005, pp. 117-218.

[11] P. de la Harpe and A. Valette, La propriété $(T)$ de Kazhdan pour les groupes localement compacts (avec un appendice de Marc Burger), Astérisque 175 (1989).

[12] G. Higman, B. H. Neumann and H. Neumann, Embedding theorems for groups, J. London Math. Soc. 24 (1949), 247-254.

[13] G. Higman and E. Scott, Existentially Closed Groups, Clarendon Press, Oxford, 1988.

[14] R. C. Lyndon and P. E. Schupp, Combinatorial group theory, Ergeb. Math. Grenz. 89, Springer, Berlin, 1977.

[15] D. A. Martin, The axiom of determinacy and reduction principles in the analytical hierarchy, Bull. Amer. Math. Soc. 74 (1968), 687-689.

[16] D. A. Martin, Borel determinacy, Ann. Math. 102 (1975), 363-371.

[17] Y. Ollivier and D. Wise, Kazhdan groups with infinite outer automorphism group, Trans. Amer. Math. Soc. 359 (2007), 1959-1976.

[18] A. Yu. Ol'shanskii, SQ-universality of hyperbolic groups (Russian), Mat. Sb. 186 (1995), 119-132; translation in Sb. Math. 186 (1995), 1199-1211.

[19] Hartley Rogers Jr., Theory of Recursive Functions and Effective Computability, 2nd edition, MIT Press, Cambridge, 1987.

[20] Y. Shalom, Rigidity of commensurators and irreducible lattices, Invent. Math. 141 (2000), $1-54$.

[21] Y. Shalom, The algebraization of Kazhdan's property (T), in Proc. Internat. Congr. of Mathematicians, Vol. II, EMS Publ. House, Zürich, 2006, pp. 1283-1310.

[22] A. A. Suslin, On the structure of special linear groups over polynomial rings, Math. USSR Izv. 11 (1977), 221-238.

[23] S. Thomas, Topological full groups of minimal subshifts and just-infinite groups, to appear in the Proceedings of the 12th Asian Logic Conference. 
[24] C. Weibel, The K-book: an introduction to algebraic K-theory, Graduate Studies in Math. vol 145, AMS, Providence, 2013.

[25] J. Williams, Universal countable Borel quasi-orders, preprint, 2012.

Mathematics Department, Rutgers University, 110 Frelinghuysen Road, Piscataway, New JeRsey 08854-8019, USA

E-mail address: sthomas@math.rutgers.edu

Department of Mathematics, California institute of Technology, Pasadena, CA 91125

E-mail address: jaywill@caltech.edu 